\title{
An Innovative Technique to Repair Cervical Oesophageal Perforation using Oro-oesophageal Tube and AMBU
}

\author{
Manish Gupta, ${ }^{1}$ Ginni Datta ${ }^{1}$
}

\section{Introduction:}

\section{ABSTRACT}

Oesophageal perforation is a rare condition and has high morbidity and mortality. Both the morbidity and mortality are directly related to the delay in diagnosis and start of treatment. Primary closure with drainage is recommended if perforation is detected in less than 24 hours. Treatment for delayed or missed rupture of oesophagus is not very clear and is controversial.

\section{Case Report:}

We hereby report a case of delayed diagnosed cervical oesophagus rupture following gunshot, which was primarily repaired. Special emphasis is on newer technique of fistula localization using oro-oesophageal tube and AMBU for better closure. The fistula closure was successful and no there were no post-operative complications.

\section{Discussion:}

The symptoms and signs on presentation of patient of cervical oesophagus perforation are discussed along with the differing treatment paradigms for early versus late presentation. Surgical repair with primary closure may be undertaken, even in late presentation of oesophagus rupture. This technique of oro-oesophageal tube used along with AMBU, may be useful in cases of both cervical and upper thoracic oesophagus rupture.

Kevwords:

Esophageal Perforation; Wounds, Gunshot

$\mathrm{O}$ esophageal perforation is an uncommon but lethal disease. Delay in diagnosis and subsequent treatment occurs in more than $50 \%$ of cases, leading to high mortality rate of $60 \%$ and if the treatment is started within 24 hours of perforation the mortality rate decreases to $10 \% .^{1,2}$ Endoscopic procedures are the most common cause of this deadly disease. ${ }^{3}$ The widespread use of both diagnostic and therapeutic endoscopy has resulted in increase in prevalence of oesophageal perforation. ${ }^{4}$ Other than iatrogenic it may be following trauma, foreign body or even spontaneous.

We present a rare case of cervical oesophagus perforation following gunshot injury, presenting late

1 - Department of ENT, Maharishi Markandeshwar Institute of Medical Science and Research, MMU, Ambala, Haryana, India.

Corresponding author:

Dr Manish Gupta

email: manishgupta1217@gmail.com with salivary leak from wound, being explored and successfully repaired using oro-oesophageal tube with artificial manual breathing unit (AMBU). The discussion includes various treatment modalities available and benefits of this innovative use of AMBU being discussed.

\section{Case Report}

A 23 year male, presented to our out- patient department, with history of gunshot injury neck 3 days prior to presentation, subsequent suturing of wound at centre close to home and complaint of saliva leak through the wound for last two days. The patient complained of pain in neck, difficulty swallowing with no difficulty in breathing. There were no sign of sepsis and on local examination there was $3 \mathrm{~cm}$ horizontal suture line on left lower lateral neck, with crepitus and salivary leak from the wound. The diluted Barium swallow with chest X-ray PA view showed extravasated luminal contrast. 


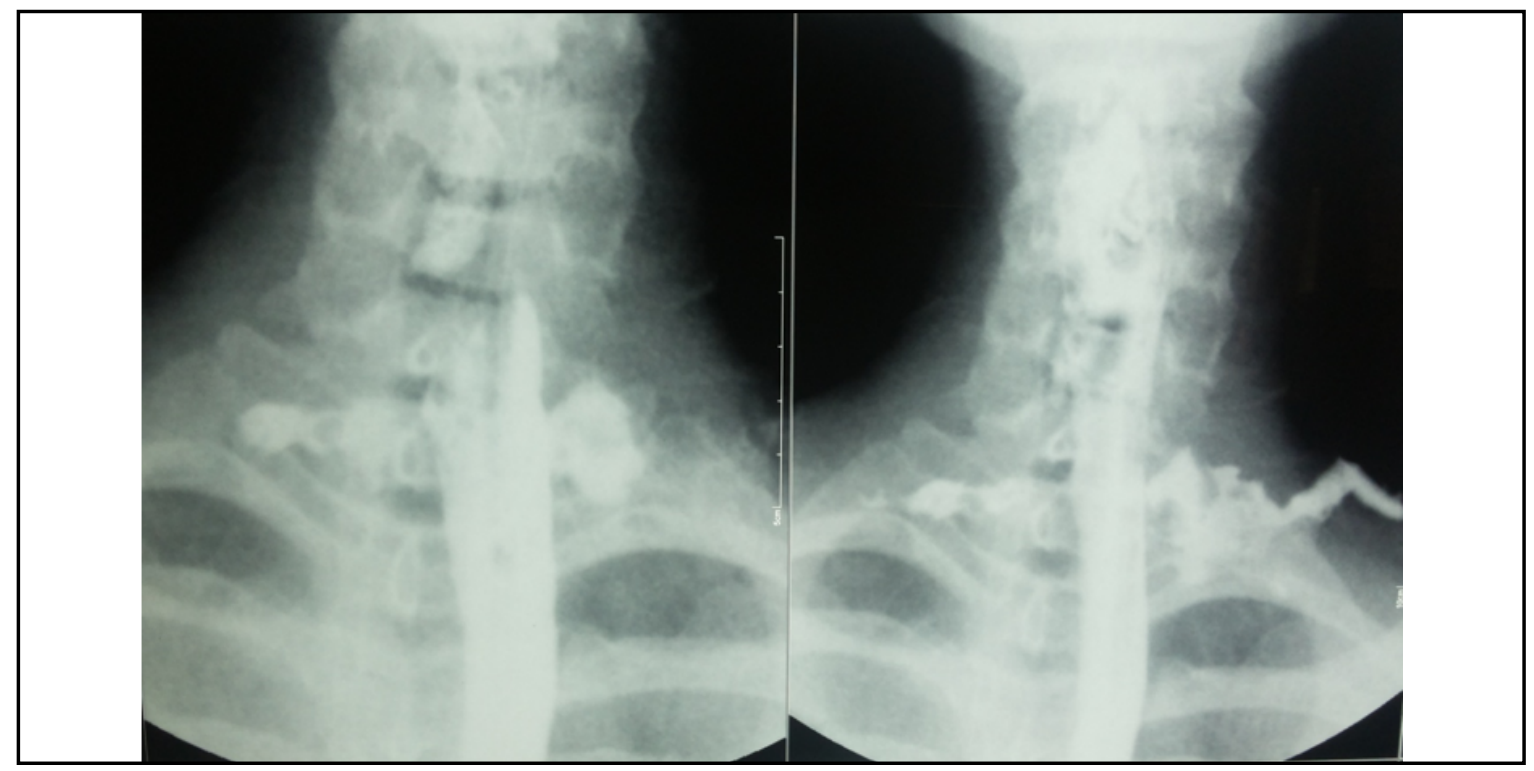

Fig.1: The preoperative diluted Barium swallow on chest x-ray PA view showing flow of luminal contrast on both sides of neck into the entry and exit wounds.

(Fig. 1) The computed tomogram with oral gastrograffin, further confirmed perioesophageal fluid collection with radiopaque dye, subcutaneous emphysema and bullet in right supraclavicular region. (Fig. 2)

The routine blood and urine examination were done and patient taken up for neck exploration under general anaesthesia. Horizontal neck crease incision was given, subplatysmal flap elevated, mucopus removed and fistula site identified in cervical oesophagus. Necrotic nonviable and grossly contaminated tissue was debrided. The technique which further helped in identification of fistula site and further in checking the adequacy of repair was devised by the main surgeon. In this, another cuffed endotracheal tube of number ${ }^{7,5}$ was placed perorally in the oesophagus and artificial manual breathing unit (AMBU) attached on its outer end. (Fig. 3)

The air was pumped in this oro-oesophageal tube with the wound filled with normal saline. The site of air bubbles in the wound confirmed the number, site and size of fistula. The fistula was repaired in two layers, first extramucosal and second muscular. The sternal fibers of sternocleidomastoid were cut, rotated and sutured over fistula site for further wound reinforcement. Intraoperatively nasogastric feeding tube was placed and following repair, it was ensured that there is no leak, from the site, by pressing air into oro-oesophageal tube. The suction drain placed and wound was closed in two layers.

Intravenous antibiotics, analgesics and nasogastric tube feeding were given for 10 days. Postoperative period was uneventful and patient discharged on oral feed. In one year follow up he has no complications like stricture or reflux.

\section{Discussion}

Perforation of oesophagus may have diverse causes, from traumatic, iatrogenic, neoplastic to foreign body and even spontaneous. ${ }^{5}$ It is usually iatrogenic, mostly following endoscopic procedures. ${ }^{6}$ Penetrating sharp injuries or gunshot can cause tissue damage that may be missed during examination, therefore high index of suspicion of oesophageal perforation is warranted in penetrating injury of neck. ${ }^{7}$

The rise in morbidity and mortality due to delayed initiation of treatment is due to unique anatomy (lack of strong serosal layer and unusual blood supply) and position of the oesophagus (proximity to vital structure). There occurs early seedling of bacteria 


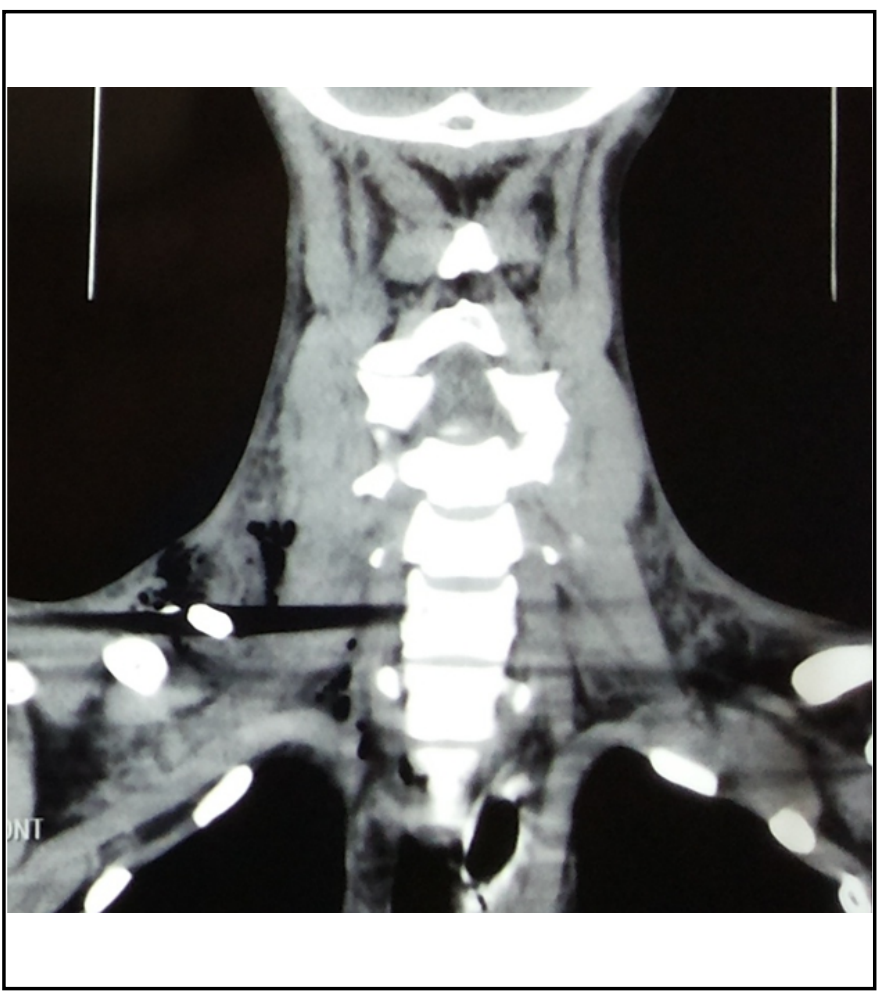

Fig.2: Coronal section of Computed Tomogram Neck showing impacted bullet in right supraclavicular region. and trickling of digestive enzymes into mediastinum, leading to mediastinitis, empyema, sepsis and multiple organ dysfunction syndrome. ${ }^{8}$ Further, the rarity of perforation and its nonspecific or diverse symptoms leads to diagnostic and treatment delay in more than $50 \%$ of perforations. ${ }^{1}$

The high risk of damage to vascular structure causing hemorrhage, airway compromise or oesophagus trauma from high velocity projectiles led Brennan et al to coin the phrase "small holes equal big pathology". 9

Presenting symptoms depend upon the site of perforation, cause and time of presentation. Pain in the neck, chest, back or epigastric region of abdomen is usually present. Other symptoms may be vomiting, hematemesis, dysphagia, tachypnea, cough and fever. ${ }^{5}$ The following signs like tachycardia, hypotension, shock, fever, subcutaneous emphysema, pneumomediastinum, pleural effusion, pneumothorax, hemothorax or collapse of the lung may be present. ${ }^{5}$ The triad of vomiting, chest pain and subcutaneous emphysema, as seen in thoracic oesophagus perforation, is known as the Mackler triad. ${ }^{10}$ This was absent in our case of cervical oesophagus perforation.

Diagnosis is confirmed by contrast leak on chest

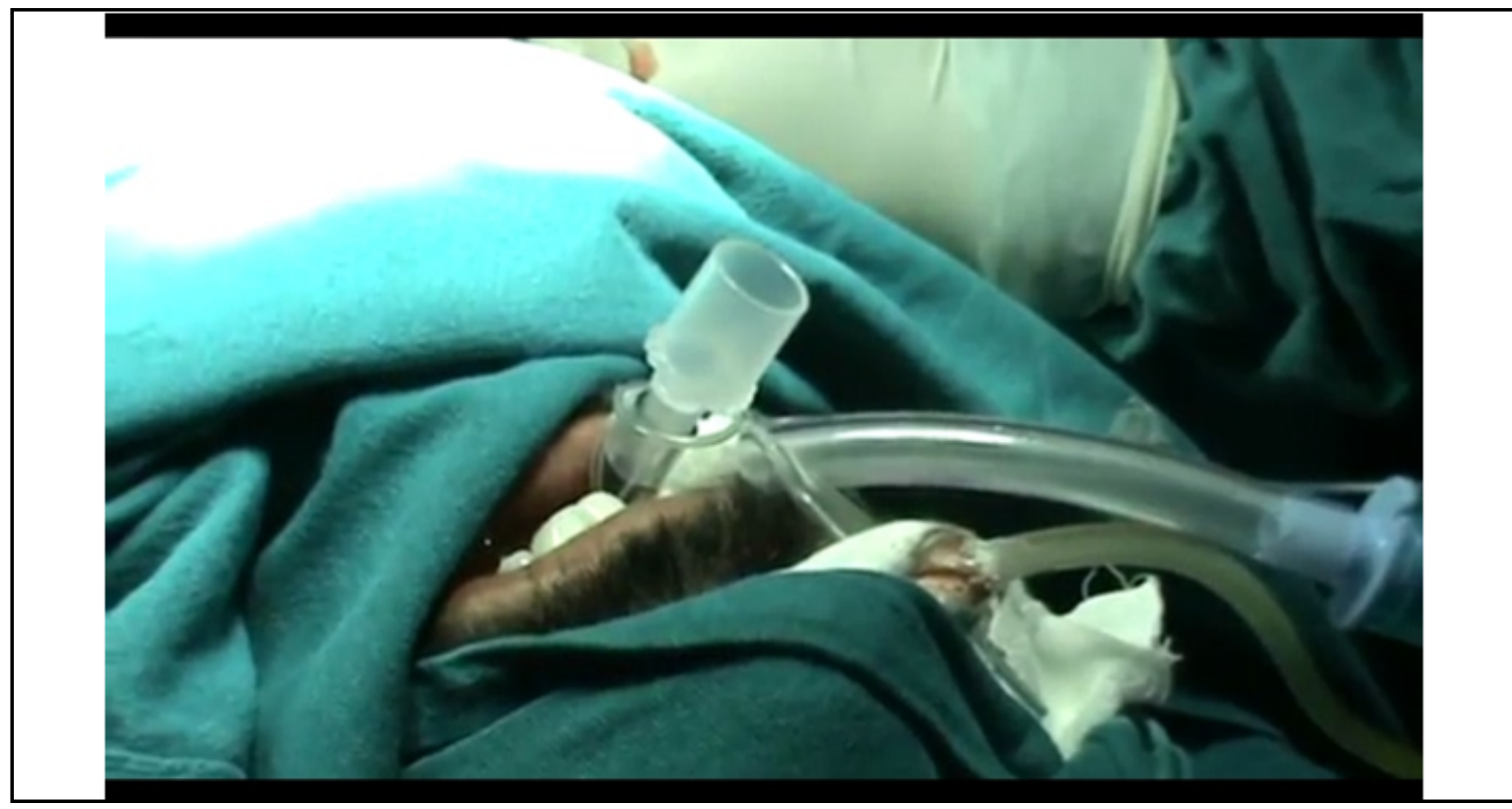

Fig.3: Intra-operative clinical picture showing two endotracheal tube placed perorally. First going in larynx and attached with ventilator while second is in the esophagus. 
radiograph using water soluble contrast medium, given per orally in most cases, similar to our case. ${ }^{11}$ Barium contrast is avoided to prevent barium related inflammation of the mediastinum, if there is perforation. Computed tomogram (CT) with oral contrast, guides regarding the exact site and size of perforation and any chest complication. There is disputed or no role of upper gastrointestinal endoscopy in diagnosis of suspected oesophageal perforation. ${ }^{12}$ Though endoscopy is helpful as a guide to the lumen for placing the nasogastric feeding tube. ${ }^{13}$

The surgical repair of oesophageal perforation had poor result until the availability of total parenteral nutrition, better antibiotics and improved surgical techniques, as available nowadays. There are several types of treatment available. The patient is immediately started on intravenous broad spectrum antibiotics, intravenous fluids or total parenteral nutrition keeping nil per oral till the decision regarding surgical primary closure or conservative management is taken.

The primary closure means detailed wide exploration of the region, identification of wound site and mucosal edges, adequate debridement of necrotic muscle and mucosa, followed by precise closure in layers, over suction drain. This is advisable in sharp injury to neck leading to oesophagus fistula or to repair perforation following previous oesophageal surgery or bullet wound of the oesophagus.

Use of oro-oesophageal tube along with AMBU will help in easy and precise localization of fistula and confirm tight closure intra-operatively. Though, it is helpful in cervical and upper thoracic oesophagus perforation detection intraoperatively, it has limited role in lower thoracic oesophagus perforations, due to limited length of the available endotracheal tube. If the perforation is diagnosed early, it may be possible to perform primary repair the procedure of choice, particularly if the remaining oesophagus is intact. ${ }^{14}$ The buttressing of suture line after primary closure, as done in our case with sternocleidomastoid muscle, provides reinforcement with vascularized tissue and decreases the risk of fistula formation. ${ }^{15}$

Another more conservative method relies mainly on drainage of wound along with nasogastric / feeding gastrostomy. This method is preferred when size of fistula is small and mostly following instrumental oesophageal perforation, following dilatation of benign strictures or for achalasia cardia. On contrary, one study had concluded that drainage alone for oesophageal perforation, whether diagnosed early or late, cannot be advocated as a safe or effective method of treatment. ${ }^{14}$

A more aggressive method is to resect the perforated portion of the oesophagus and perform primary anastomosis. This is preferred in patients with benign but difficult to dilate strictures and with carcinoma.

There is anothermethod which relies on diversion of the oesophagus, with side or end cervical oesophagostomy. This is useful in patients with carcinoma and is followed by radiotherapy.

Indwelling Mousseau-Barbin tube or endoluminal naso-oesophageal tube may also be used for tracheaoesophageal fistula, in malignant cases, to close off the fistula. Fibrin sealant may also be used in treatment of small oesophageal perforations, applied endoscopically from lumen. ${ }^{16}$ This is useful only in perforation size less than $1.5 \mathrm{~cm}$, with clean edges and minimal or no symptoms of infection.

In a study over 47 patients with oesophagus perforation, the patients receiving treatment within first 24 hours had greater success rate, full recovery without complication, as compared to those who received treatment more than 24 hours after perforation. ${ }^{14}$ The study concluded that timing of intervention plays important role in managing oesophagus perforation.

Recent literature favors primary repair of delayed diagnosed oesophagus perforation as the best surgical approach with consistently low mortality ranging from $3 \%$ to $13 \%$. ${ }^{17}$ Thus, no patient should be deprived from surgical repair due to delayed diagnosis.

Recently advances in biomaterial gives new generation of stents which have non-permeable covering and apply radial force sufficient to occlude a transmural oesophageal injury. ${ }^{18}$ This is considered safe and is less invasive treatment route compared to direct primary repair. ${ }^{18}$ Despite these benefits, acute cervical oesophageal injuries are better repaired operatively, as most patients are unable to tolerate a stent that lies proximal to the cricopharyngeus muscle. ${ }^{18}$ 


\section{References}

1. Bladergroen MR, Lowe JE, Postlethwait RW. Diagnosis and recommended management of esophageal perforation and rupture. Ann Thorac Surg. 1986; 42(3):235-9

2. Sarr M G, Pemberton J H, Payne W S. Management of instrumental perforations of the esophagus. J Thorac Cardiovasc Surg. 1982; 84(2):211-8

3. Okten I, Cangir AK, Ozdemir N, Kavukcu S, Akay H, Yavuzer S. Management of esophageal perforation. Surg Today 2001; 31(1):36-9

4. Bresadola V, Terrosu G, Favero A, Cattin F, Cherchi V, Adani GL, Marcellino MG, Bresadola F, De Anna D.. Treatment of perforation in the healthy esophagus: analysis of 12 cases. Langenbeck's Arch Surg. 2008; 393(2):135-40

5. Kaman L, Iqbal J, Kundil B, Kochhar R. Management of esophageal perforation in adults. Gastroenterol Res. 2010; 3(6):235-44

6. Merchea A, Cullinane DC, Sawyer MD, Iqbal CW, Baron TH, Wigle D, Starr MG, Zielinski MD. Esophagogastroduodenoscopy-associated gastrointestinal perforations: a single center experience. Surgery 2010; 148:876-80, discussion $881-2$

7. Enguidanos L, Pfleiderer A, Smith W, Ramkumar S. Pneumomediastinum secondary to an apparently trivial stab wound to the neck: the value of the Hamman's sign and thorough radiological investigation. Emerg Med J. 2005; 22:230-1

8. Altorjay A, Kiss J, Voros A, Sziranyi E. The role of esophagectomy in the management of esophageal perforations. Ann Thorac Surg. 1998; 65(5):1433-6
9. Brennan J, Gibbons MD, Lopez M, Hayes D, Faulkner J, Eller $\mathrm{RL}$, Barton C. Traumatic airway management in operation Iraqi freedom. Otolaryngol Head Neck Surg. 2011; 144:376-80

10. Mackler SA. Spontaneous rupture of the esophagus; an experimental and clinical study. Surg Gynecol Obstet. 1952; 95:345-56

11. Vial CM, Whyte RI. Boerhaave's syndrome: diagnosis and treatment. Surg Clin North Am. 2005; 85:515-24

12. Arantes V, Campolina C, Valerio SH, de Sa RN, Toledo C, Ferrari TA, Coelho LG. Flexible esophagoscopy as a diagnostic tool for traumatic esophageal injuries. J Trauma 2009; 66:167782

13. Soong $\mathrm{Wj}$. Endoscopic diagnosis and management of iatrogenic cervical esophageal perforation in extremely premature infants. J Chin Med Assoc. 2007; 70:171-5

14. Skinner DB, Little AG, DeMeester TR. Management of esophageal perforation. The Am J Surg. 1980; 139:760-4

15. Gouge TH, Depan HJ, Spencer FC. Experience with the Grillo pleural wrap procedure in 18 patients with perforation of the thoracic esophagus. Ann Surg. 1989; 209(5):612-7; discussion $617-9$

16. Harries K, Masoud A, Brown TH, Richards DG. Endoscopic placement of fibrin sealant as a treatment for a long-standing Boerhaave's fistula. Dis Esophagus 2004; 17(4):348-50

17. Wu JT, Mattox KL, Wall MJ, Jr. Esophageal perforations: new perspectives and treatment paradigms. J Trauma 2007; 63(5):1173-84

18. Ong GK, Freeman RK. Endoscopic management of esophageal leaks. J Thorac Dis. 2017; 9(Suppl 2):S135-S145 